\title{
Antibiotics: Need for new fences", a prospective utilization evaluation of cephalosporin's and its combination in tertiary care referral hospital
}

\author{
Dilip Chandrasekhar ${ }^{\mathrm{a}, *}$, Jaffer Chalil Parambil ${ }^{\mathrm{b}}$, P.B. Chinnu ${ }^{\mathrm{a}}$, Cyriac T. Mundadan ${ }^{\mathrm{a}}$, \\ Danisha Pattani ${ }^{\mathrm{a}}$ \\ a Department of Pharmacy Practice, Al Shifa College of Pharmacy, Kizhattur, Perinthalmanna, Malappuram, Kerala, 679325, India \\ ${ }^{\mathrm{b}}$ Consultant Physician, Dept of General Medicine, KIMS Alshifa Hospital Pvt Ltd, Malappuram, Kerala, India
}

A R T I C L E I N F O

\section{Keywords:}

Cephalosporin

Antibiotics

Utilization

\begin{abstract}
A B S T R A C T
Background: The cephalosporins are a large group of related $\beta$-lactam antimicrobial agents with broad spectrum of activity. Drug Utilization and Evaluation (DUE) is an ongoing, structured process to analyse the pattern of drug administration in various practice settings, including hospitals in relation to guidelines or predetermined standards thereby promoting the appropriate and effective use of drugs.

Methods: This prospective interventional study was conducted for a duration of six months among the in-patients of various departments of a multispecialty hospital located in Kerala, India. Cephalosporins were evaluated. Results: Study conducted over a period of 6 months revealed that most of the therapy was definite $45 \%$ $(n=120)$ followed by empirical $32 \%(n=85)$ and the least type was prophylactic $23 \%(n=62)\left(x_{2}=16.965\right.$, d.f $=2, p<0.0010$ ). Inappropriate use of cephalosporins were noted in 30 out of 267 cases (11.2\%). Among these majority $93 \%(n=28)$ were wrong drug selection followed with wrong days of therapy and failure of deescalation, $3.33 \%(n=1)$ each. In $13 \%(n=8)$ cases, drug was given beyond the actual duration. De-escalation was not done in $4 \%(n=3)$ cases and in $8 \%(n=5)$ cases the treatment was done against the culture and sensitivity pattern. The most common drug used in combination with cephalosporins was metronidazole (36\%). Conclusion: Extensive use of third generation cephalosporin was observed in the study with facts and figures revealing their inappropriate use. This climaxes the need of continuous educational intervention by clinical pharmacist along with the modification of hospital antibiotic policy.
\end{abstract}

\section{Introduction}

In developing countries, infectious diseases are the most common causes of morbidity and mortality. The use of antibiotics has contributed to the dramatic fall in morbidity and mortality from communicable and infectious disease over the last 50 years globally. However, the control of infectious disease is seriously threatened by the steady increase in the number of microorganisms that are resistant to antimicrobial agents. Emergence of antimicrobial resistance is a result of the use, over use and misuse of antibiotics. When considering this, the logical first step is to evaluate the suitability of antibiotic usage. ${ }^{1,}{ }^{3}$ The Cephalosporins are a large group of related $\beta$-lactam antimicrobial agents with broad spectrum of activity, low rates of toxicity and ease of administration. Various Cephalosporins are effective for treatment of many conditions, including pneumonia, skin and soft tissue infections, bacteraemia and meningitis. These antimicrobials are also among the most expensive. Cephalosporins are grouped based on their antibacterial properties and when they were introduced: First generation cephalosporins include cephalexin and cefazolin. They have good activity against a wide spectrum of gram-positive bacteria including penicillinase-producing staphylococci. ${ }^{9}$ Second generation cephalosporins include cefaclor, cefuroxime and cefoxitin. They are more stable to hydrolysis by beta-lactamases produced by gram negative bacteria. Ceftriaxone is a third generation cephalosporin. They have the widest spectrum of activity compared to other generations of cephalosporins and are active against gram-negative organisms, including many of the significant enterobacteriaceae. They are also very active against streptococci. ${ }^{7}$ Overprescribing, misuse of antibiotics and low adherence to treatment guidelines were seen in both public and private settings. ${ }^{8} \mathrm{~A}$ multidisciplinary approach including supervision of their use by infectious disease consultants and/or clinical pharmacists, provision of continuing education regarding appropriate antimicrobial drug use, and implementation of automatic stop orders is warranted. Another method increasingly used in this era of cost constraints and quality

\footnotetext{
* Corresponding author. Department of Pharmacy Practice, Al Shifa College Of Pharmacy, Poonthavanam P.O Perinthalmanna, Kerala, 679325, India.

E-mail address: dillu7@gmail.com (D. Chandrasekhar).
} 
assurance is drug utilization evaluation (DUE).This tool was adapted by pharmacists to assess appropriateness of usage of various medications. The purpose of a DUE is generally to detect possible problems with, and improve drug use. ${ }^{9}$ Drug utilization has been defined as 'the marketing, distribution, prescription and use of drugs in a society, with special emphasis on the resulting medical, social and economic consequences (WHO Expert Committee, 1977).The concept of the drug use review (DUR) was raised by the Joint Commission on Accreditation of Healthcare Organizations (JCAHO). Subsequently, the drug use evaluation (DUE) was developed on the basis of the DUR. DUE is an ongoing, structured process to analyse the pattern of drug administration in various practice settings, including hospitals in relation to guidelines or predetermined standards thereby promoting the appropriate and effective use of drugs. DUR is classified in three categories: DUR is typically classified in three different categories: prospective, concurrent and retrospective. Prospective review involves evaluating a patient's planned drug therapy before a medication is dispensed. This process allows the pharmacist to identify and resolve problems before the patient has received the medication. Pharmacists routinely perform prospective reviews in their daily practice by assessing a prescription medications dosage and directions while reviewing patient information for possible drug interactions or duplicate therapy. Issues commonly addressed by prospective DUR includes, clinical abuse/misuse, drugdisease contraindications, drug dosage modification, drug-drug interactions, drug-patient precautions, formulary substitutions, inappropriate duration of drug treatment. Concurrent review is performed during the course of treatment and involves the on-going monitoring of drug therapy to foster positive patient outcomes. This type of review allows therapy for a patient to be altered if necessary. A retrospective DUR reviews drug therapy after the patient has received the medication. A retrospective review aims to detect patterns in prescribing, dispensing or administering drugs. Pharmacists can then, in collaboration with prescribers and other members of the health care team, initiate action to improve drug therapy for patients. ${ }^{12}$ DUE has a seven-steps procedure, comprising of establishing responsibility, developing the scope of activities and defining the objectives, establishing criteria for review of the medicine, data collection, data analysis, feedback to the prescribers and making a plan of action and follow up. ${ }^{10}$ The organizational body (e.g., quality management committee, pharmacy and therapeutics committee) responsible for the DUE process should have, at a minimum, prescriber, pharmacist, nurse, and administrator representation. Pharmacists, by virtue of their expertise and their mission of ensuring proper medication use, should exert leadership and work collaboratively with other members of the health care team in the on-going process of medication-use evaluation and improvement.

\section{Materials and methods}

The prospective interventional study was conducted for duration of six months among the in-patients of various departments of a multispecialty hospital located in Kerala, India. The study was conducted among the various departments including the General Medicine I and II, pulmonology, General Surgery considering the extensive use of antibiotic in the corresponding departments. The prospective method mainly involves evaluating a patient's planned drug therapy before a medication is dispensed. The data for the study was taken from case sheets, treatment charts, and personal interview with the patient, laboratory data, discharge cards/advice on discharge. A form for data collection was developed after assessing different standard forms. All inpatient records irrespective of any age group of General Medicine 1, General Medicine 2, General Surgery and Pulmonology containing cephalosporins and its combinations were included in the study. Out patient records of the selected departments, all outpatient and inpatient records of other departments and all cases of pregnancy and lactation were excluded. Relevant data were collected during ward rounds and patients were directly interviewed in order to report any specific ADRs like diarrhea due to the antibiotic therapy. ANTIBIOTIC POLICY 2014-2015, Guidelines for the use of antibiotics and other anti-infectives, which was developed by the Department of hospital infection control, for ensuring the judicious use of the antibiotics was set as the guideline for monitoring the drug use. Ethics committee clearance was obtained from the hospital ethical committee prior to the conduct of study. The study was conducted in four phases. Phase I was collection of patient data and assessment. The complete details of all the cephalosporin therapy cases were entered into a self-designed data collection form. All the enrolled patients were monitored from the date of admission until the last day of antibiotic therapy. Criteria for evaluation includes the appropriateness of drug of choice for indication, dose, frequency of the drug administration, duration of the therapy, drug interactions and adverse drug reactions of cephalosporins were evaluated. The study was initiated with the null hypothesis that there exists an appropriate selection of antibiotics for treatment and prophylaxis. Sample size for the study was set at 267 by assuming the prevalence ratio as $50 \%$ at confidence interval of $95 \%$. For assessing the significance of age, mean and standard deviation was used. The key point of the study, appropriateness of drug selection was analyzed by using the Chi square test - test of goodness of ratio. The entire test was conducted at a confidence interval of $95 \%$. Evaluation of prescriptions was done in phase 2 . The entire data was analyzed by using different statistical methods in consultation with bio statistician. Once the collected data was analyzed as per the statistical test, the level of appropriateness was established. The various scopes for the improvement of the drug therapy were pointed out. Recommendations for improving the quality of hospital antibiotic policy were also suggested. Following the intervention, the study was continued for a short period for accessing the effectiveness which was the fourth phase.

\section{Results}

Study conducted over a period of 6 months revealed that male 137 (51.32\%) predominated over females $130(49.68 \%)$ in total number of data collected. Most of the therapy was definite $45 \%(n=120)$ followed by empirical $32 \%(n=85)$ and least type was prophylactic $23 \%$ $(\mathrm{n}=62)\left(x_{2}=16.965\right.$, d.f $\left.=2, \mathrm{p}<0.0010\right)$. It was observed that the maximum number of cases were reported from age group 71-80yrs (35.96\%), followed by $91-100 y r s(18.74 \%)$ and $41-50$ yrs (15.73\%). Cases of the type B ADR was significantly greater than that of type A $\left({ }^{x} 2=26.00\right.$, d.f $\left.=1, p<0.001\right)$. Pooled data for all four departments showed that about $60 \%(\mathrm{n}=120)\left({ }^{x} 2=7.257\right.$, d.f $\left.=1, \mathrm{p}<0.01\right)$ drug selections were not according to the guidelines and in remaining $40 \%(n=107)$ cases drug selection was as per guidelines. Wrong drug selection was done in 65.93\% $(\mathrm{n}=89)\left(\mathrm{d} . \mathrm{f}=2, \mathrm{p}<0.01,{ }^{\times} 2=47.8\right.$ ) cases, while in case of wrong days it were $6.667 \%(\mathrm{n}=9)$. De-escalation was not done in $2.96 \%(n=4)$ cases, in $12.59 \%(n=17)$ cases treatment was against the culture and sensitivity report. This is depicted in Fig. 1. In 30 cases, there was inappropriateness. In $93 \%$ $(n=28)$ cases there was wrong drug selection. Wrong days of therapy and de-escalation was there in $3.33 \%(\mathrm{n}=1)$ cases each. 62 inappropriate cases were form GM II $\left(x_{2}=30.356\right.$, d.f $\left.=3, \mathrm{p}<0.001\right)$, $44 \%(n=27)$ cases was due to wrong drug selection, followed by wrong frequency in $17 \%(n=10)$ cases. In $13 \%(n=8)$ cases, drug was given beyond the actual duration. De-escalation was not done in $4 \%$ $(n=3)$ cases and in $8 \%(n=5)$ cases the treatment was done against the culture and sensitivity pattern. Out of the total of 29 cases, 16 cases were inappropriate in pulmonology. $62 \%$ cases of wrong drug selection and $19 \%(n=3)$ of wrong dose were found. Wrong frequency was followed in $13 \%(n=2)$ cases and $6 \%(n=1)$ inappropriateness in number of days. Appropriate drug cases were significantly higher in the department of general surgery compared to the other departments $\left({ }^{\times} 2=9.528\right.$, d.f $\left.=3, p<0.05\right) .41$ cases of inappropriate prescriptions was found, in that $59 \%(n=24)$ were due to wrong drug selection 


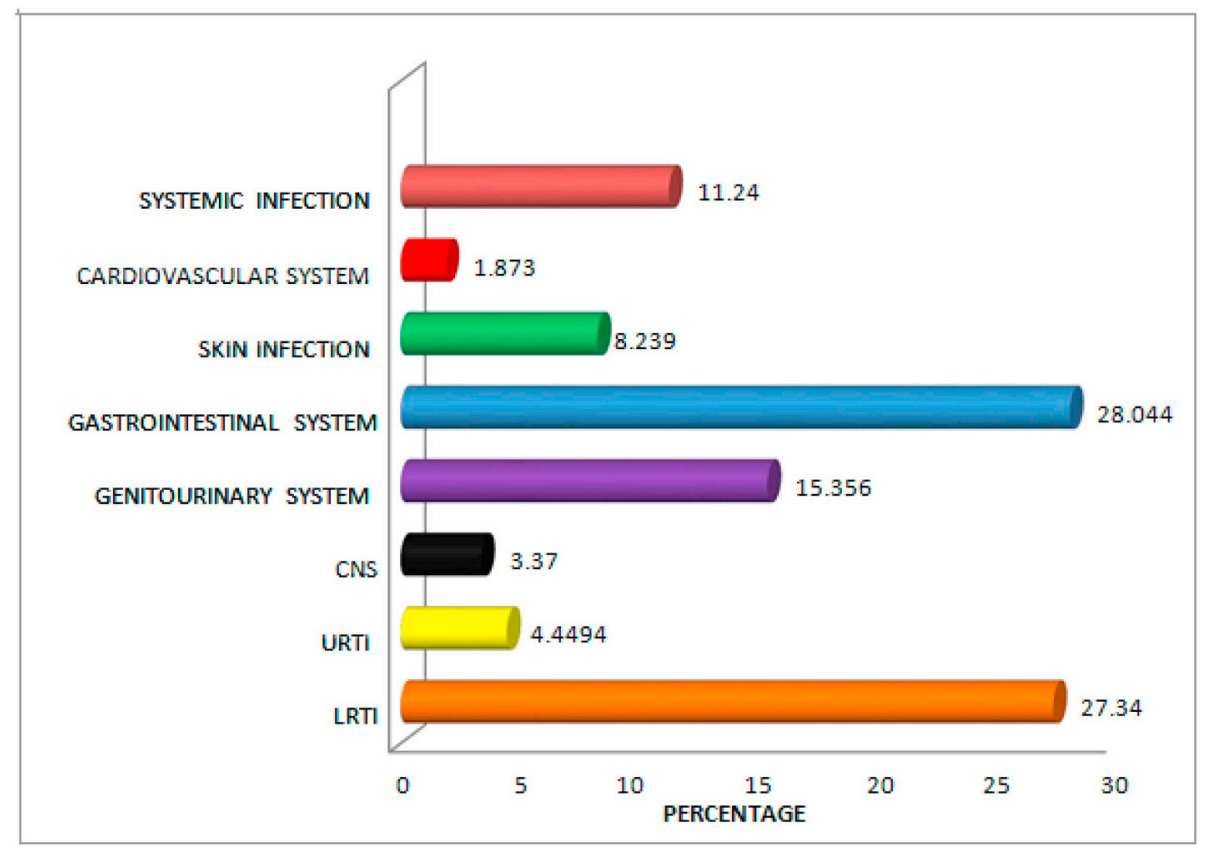

Fig. 1. Reasons for inappropriateness of cephalosporin therapy.

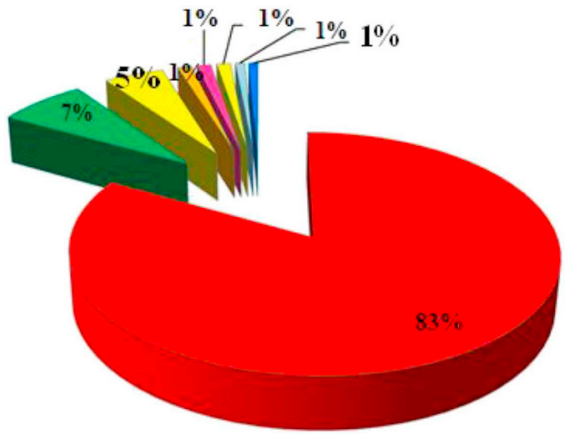

口CEFOPERAZONE

III CEFAZOLIN

III CEFTRIAXONE

III CEFIXIME

IITEFOTAXIME

ПCEFUROXIME

ПCEFEPIME

MEFTAZIDIME

Fig. 2. Individual drug therapy.

and $29 \%(\mathrm{n}=12)$ due to wrong dose. Cases of concomitant antibiotics use were significantly higher in the department of general medicine 2 compared to other departments $\left(x_{2}=27.364\right.$, d.f $\left.=3, p<0.001\right)$. Among the antibiotics, metronidazole (36\%) was widely used especially in general surgery followed by moxifloxacin (32\%), While levofloxacin covers $11 \%$ of the total. Tazobactam was used in $10 \%$ of cases while azithromycin was used in $7 \%$. Both meropenem and rifampicin were used in $2 \%$ of cases. From Fig. 2, it was observed that major drug used among cephalosporins was cefoperazone $83 \%$, others includes cefazolin $7 \%$, ceftriaxone $5 \%$, cefixime $1 \%$, cefotaxime $1 \%$, cefuroxime $1 \%$, cefepime $1 \%$, ceftazidime $1 \%$. In $97 \%$ of cases the condition was treated with a single cephalosporin while in the remaining $3 \%$ of cases two cephalosporins were used. $28.044 \%$ of antibiotics were prescribed for gastrointestinal system diseases, followed by LRTI (27.34\%). Least number of indications were for cardiovascular system $1.873 \%$. (Fig. 3). Cases of definite therapy is significantly higher than the empiric therapy $\left(x_{2}=4.484\right.$, d.f $\left.=1, p<0.05\right)$.In the department of general medicine 1 no significant difference could be detected in the number of cases between definite and empirical therapy $\left({ }^{\chi} 2=1.524\right.$, d.f $=1$, $\mathrm{p}<0.05$ )In the department of general surgery prophylactic cases were significantly higher than definite and empirical cases $\left(. x_{2}=59.786\right.$, d.f $=2, \mathrm{p}<0.001$ ). In the pulmonology department, cases of the definite therapy is significantly higher than the empirical therapy $\left({ }^{x} 2=4.172\right.$, d.f $\left.1=, p<0.001\right)$.

\section{Discussion}

Drug utilization studies are obliging in understanding the contemporary practice in clinical settings. The study revealed that rationality was lacking as there was excessive use of the above mentioned classes of antibiotics. This study provides the data on the nature and extent of usage of cephalosporins in patients admitted to different departments of the hospital. A total of 267 cephalosporin's prescribed patients are identified and included in this study. When the findings of Shekar et $\mathrm{a}^{14}$ shows a male population of $61.6 \%$, number of females appeared to be higher in the current study. This can be better elucidated by the backup using the latest census report of the Malabar region. The settlement of adult male of this region in exploration of jobs has a great contribution. Extended life expectancy due to the high standard of living of this region play a part in counting majority of the included patient under the age group of 71-80, followed by $91-100$ years. On statistical evaluation, cases of definite type of the therapy is significantly higher than empirical and prophylactic, which is contradictory to the result of Jyothi et al., ${ }^{5}$ with most of the therapy being empirical. Particularly 3rd generation drugs were used in most of patients than the other generations. The prophylactic use of newer, broad spectrum drugs that are frontline therapeutic agents is generally discouraged because of the concern that widespread use will promote the emergence and spread of bacterial strains that are resistant to them. Among the four departments, the pattern of antibiotic prescribing is absolutely poles apart. This underscores the inter-individual differences of the physicians prescribing pattern, which is a main aim of our study. Multiple antibiotic treatments are generally opted under condition of sub therapeutic response or even in cases of super infection. Among the antibiotics, metronidazole (36\%) was widely used especially in General Surgery followed by moxifloxacin (32\%). This prescribing pattern was also shown in the study conducted by Shekar et al., ${ }^{4}$ and Gururaja et al. 3. In all the studies, metronidazole was the most widely using concomitant antibiotic. In Paul. $\mathrm{R}^{17}$ et al. observation also metronidazole was the mostly used antibiotic co-prescribed with cephalosporins. The wide co-prescription of metronidazole and cephalosporins maybe because metronidazole is active against gram negative anaerobes, possess good tissue penetration, extends rapid bacterial killing, its availability in oral dosage forms and economic feasibility. Conflicting outcomes presented by Jyothi et $\mathrm{al}^{5}$ showed wide use of nitroimidazoles. 


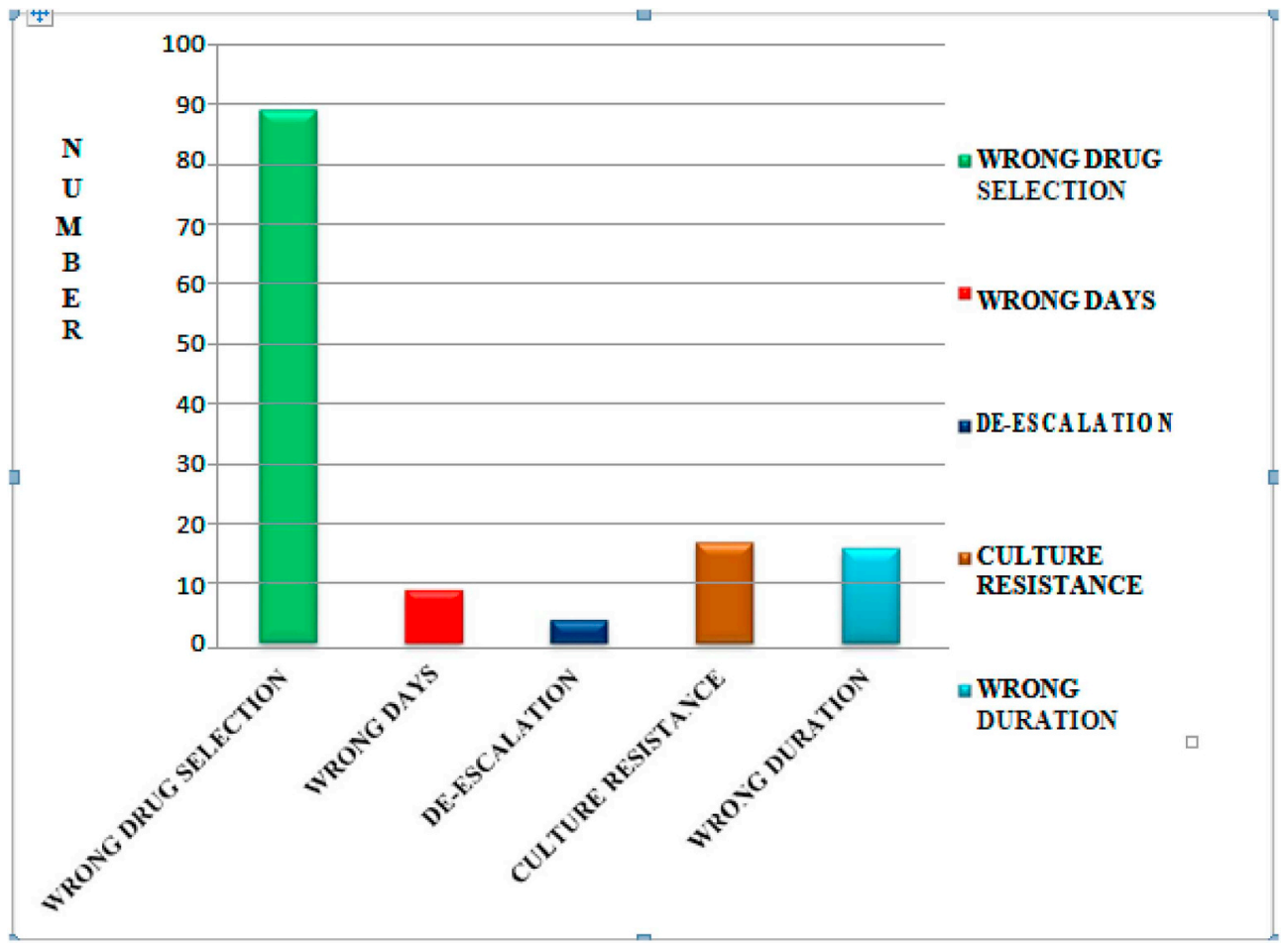

Fig. 3. Indications of cephalosporins.

Combination therapy among the cephalosporins is not generally supported by the guidelines, but in $3 \%$ of the cases, comparable to findings of Jyothi.k et al., ${ }^{5}$ two different types of cephalosporins were used. This was done when were a lower generation cephalosporin was not producing the desired effect, hence switching to a higher one. Third generation cephalosporins were used in $85 \%$ of cases analogues to Bushra et al. ${ }^{15}$ This extensive use of third generation cephalosporin may be due to its extended spectrum of activity against most of the bacteria, especially against gram negative bacteria when compared to other class/generation of cephalosporins. The Jonathan et al. ${ }^{18}$ study also presented a higher usage of third generation of cephalosporins which was akin to our results. Cefoperazone was the most widely used among the cephalosporins which may be due to the broad spectrum of activity, excellent penetration to the body tissues and wide coverage to all bacteria. On the other hand in Getasew et al. ${ }^{10}$ work, ceftriaxone was widely used due to its sensitivity pattern and lower incidence of ADR. Incidence of ADR in the study patients was found to be $11.985 \%$. ADR was detected with third generation cephalosporins than with second generation cephalosporins. This is due to the fact that cephalosporins have a higher incidence of $>2 \%$ in causing GI disorders. ADRs commonly seen in the study patients was diarrhea, this result was similar to the study conducted by Jyothi.k et al. ${ }^{5}$ which showed both GI and skin reactions. In the departments of General Medicine 2 and 1, inappropriate drug usewere about $62 \%$ and $71.429 \%$ respectively. Erroneous drug selection was the core reason behind this. Despite the fact, guidelines were suggesting antibiotics like amoxicillin and clavulanic acid, ciprofloxacin, and levofloxacin as the drug of choice for the treatment of disease like acute otitis media, acute sinusitis and enteric fever respectively, but in practical settings, cephalosporins were used. In many cases the dose given was different from that endorsed by the guideline. There were cases in which cephalosporin use were sustained in spite of a paradoxical culture report. The conclusion of Jyothi. $\mathrm{k}^{5}$ was also backing our conclusions. Owing to the broad spectrum activity of cephalosporins, they are widely used for prophylaxis in the department of General Surgery. Relating to other departments appropriate drug use was significantly higher in the department of General Surgery. Prophylaxis was the main intention of practice. Cefoperazone was extensively used. Ceftriaxone, an alternative third generation cephalosporin was widely found to be used in Poonam' ${ }^{16}$ work. On the word of Gujaral et al., ${ }^{3}$ third-generation cephalosporins were prescribed to $87.79 \%$ of patients for surgical prophylaxis which was inappropriate, and incongruous towards our outcomes. For surgical prophylaxis it was focal to select an antibiotic with narrowest antibacterial spectrum to reduce the emergence of resistance, as well broad spectrum antibiotics could be required later if patient developed serious sepsis. For that reason it was acclaimed that use of third-generation cephalosporins could be avoided in surgical prophylaxis. However being expensive, cephalosporins were used in Surgery ward to a greater extent for prophylaxis due to their excellent activity against most of the bacteria causing post-operative wound infection. When it comes to department of pulmonology, cases of the definite therapy are significantly higher than the empirical therapy. Most of the antibiotic selection was in accordance to the culture and sensitivity reports. The hospital antibiotic policy is too concise, covering only a narrow spectrum of diseases. This climaxes the need of continuing educational intervention by clinical pharmacist along with the modification of hospital antibiotic policy.

\section{Conclusion}

The inappropriate use of third generation cephalosporins for definite therapy was shown extensively in this study. Scientific reports have proved that widespread use of cephalosporins may lead to decreasing efficacy of other beta lactam ring antibiotics by forming bacterial resistance. Thus, healthcare professionals must be cautious so as to prevent clinical consequences like treatment failure, recurrence or secondary infections.

\section{Limitations}

The study was conducted as a part of regular academic program and hence the duration was limited to a period of six months. Causality assessments were not done for the reported ADRs. There are chances of bias in ADR reporting as they were reported after patient interviews. Patients being uncertain about ADRs may wrongly interpret a disease 
symptom and an adverse effect of a drug. The study was confined to a single hospital, and limited number of cases by which a generalization of utilization of cephalosporins in a large population cannot be done. Yet, the study brought out the inappropriateness in cephalosporin therapy and focus on the necessitation of rational drug use.

\section{Funding}

The authors received no financial support for the research, authorship, for the publication of this article.

\section{Conflicts of interest}

No potential conflicts of interests relevant to this article was reported.

\section{Appendix A. Supplementary data}

Supplementary data to this article can be found online at https:// doi.org/10.1016/j.cegh.2019.05.007.

\section{References}

3. Gururaja MP, et al. Cephalosporin utilization evaluation in a university teaching hospital: a prospective study. J Drug Deliv Ther. 2013;3(2):83-87.

4. Shekar HS, et al. Drug utilization and evaluation of Cephalosporin's at tertiary care teaching hospital. Int J Res Pharm Biomed Sci. 2013;4(4).

5. Jyothi K, et al. Drug utilization evaluation of cephalosporins in general medicine unit of rural tertiary care hospital. Int J Curr Pharm Res. 2012;4(2).

7. Evaluation of the use of cephalosporin antibiotics in pediatrics. RJPBCS. 2012;3(3):640

8. Abebe FA, et al. Drug use evaluation of ceftriaxone: the case of ayder referral hospital, mekelle, Ethiopia. Int J Pharm Sci Res. 2012;3(7):2191-2195.

9. Mohammadi M, et al. Drug use evaluation of three widely prescribed antibiotics in a teaching hospital in east of Ethiopia. Iran. J Pharm Care. 2013;1(3):100-103.

10. Ayinalem Getasew Amogne, et al. Drug use evaluation of ceftriaxone in medical ward of dessie referral hospital, north east Ethiopia. Int J Chem Nat Sci. 2013;1(1):12-16

12. Khan Fahmi Yousef, et al. Evaluation of the use of piperacillin/tazobactam (tazocin) at hamad general hospital, Qatar: are there unjustified prescriptions. Infect Drug Resist. 2012;5:17-21.

14. Helena Gama. Drug utilization studies. ArquiMed. 2008;22(2/3):69-74.

15. Riaz Bushra. Evaluation of the use of cephalosporin antibiotics in pediatrics. $J$ Appl Pharm Sci. April 2013;03(04):063-066.

16. takhar Poonam. Investigation of antibiotic usage pattern: a prospective drug utilization review. Der Pharm Lett. 2011;3(5):301-306.

17. Paul R. A prospective study on usage of cephalosporin's in hospitalized patients. IJPIS J Hospital Clin Pharm. 2011;1(5):1-8.

18. Jonathan GA. Ceftriaxone and cefotaxime use in victorian hospitals. Med J Aust. 2002; 176:524-529. 